\title{
Management of stage IIIA non-small-cell lung cancer: role of surgery
}

\author{
Niki Iranpour ${ }^{1}$, Jacqueline K. Olive ${ }^{1}$, Mara B. Antonoff ${ }^{2}$ \\ ${ }^{1}$ Division of Cardiothoracic Surgery, Michael E. DeBakey Department of Surgery, Baylor College of Medicine, Houston, TX, USA; ${ }^{2}$ Department of \\ Thoracic and Cardiovascular Surgery, University of Texas MD Anderson Cancer Center, Houston, TX, USA \\ Contributions: (I) Conception and design: All authors; (II) Administrative support: MB Antonoff; (III) Provision of study materials or patients: None; \\ (IV) Collection and assembly of data: N Iranpour, JK Olive; (V) Data analysis and interpretation: N Iranpour, JK Olive; (VI) Manuscript writing: All \\ authors; (VII) Final approval of manuscript: All authors. \\ Correspondence to: Mara B. Antonoff, MD, FACS. Department of Thoracic and Cardiovascular Surgery, University of Texas MD Anderson Cancer \\ Center, 1400 Pressler St, Unit 1489, Houston, TX 77030, USA. Email: mbantonoff@mdanderson.org.
}

\begin{abstract}
Controversy has long surrounded the indications for surgical resection as a treatment modality for stage IIIA non-small cell lung cancer (NSCLC). The variability in treatment decision-making results from the inclusion of a vastly heterogenous population of disease with wide-ranging presentation that is even more evident in the N2 disease subpopulation. Although several major trials have been conducted, determining surgical candidacy and prognosis of stage IIIA NSCLC remains a challenge for the multidisciplinary team. We reviewed the PubMed literature surrounding multi-modal therapy for stage IIIA NSCLC in order to determine best practices and areas in need of further development. While findings of the included studies vary, many show the addition of surgical resection to a regimen of chemotherapy and radiation therapy to improve overall survival rates. There is no consensus across studies in regard to the optimal extent and combination of the available modalities. Patient-related and tumor-related characteristics have been shown to significantly impact surgical outcomes. Survival rates are also dependent on the type of resection performed, with a lobectomy being the operation of choice when tolerable. There has been a shift in practice to using a video-assisted approach over thoracotomy that has shown preliminary improvements in survival as well. Surgery often remains a necessary component of treatment of stage IIIA NSCLC and has demonstrated improved survival rates. We recommend further investigation of long-term outcomes from multi-modal therapy in addition to the ongoing development of standardized definitions of stage IIIA disease and treatment. The role of surgery must also be reimagined with new and evolving treatment modalities in mind.
\end{abstract}

Keywords: Non-small cell lung cancer (NSCLC); stage IIIA NSCLC; lung cancer; thoracic surgery; multimodality therapy

Received: 26 March 2020; Accepted: 24 June 2020; Published: 25 August 2021.

doi: $10.21037 /$ ccts-20-65

View this article at: http://dx.doi.org/10.21037/ccts-20-65

\section{The present role of surgery}

Controversy has long surrounded the indication of surgical resection as a treatment modality for stage IIIA non-small cell lung cancer (NSCLC). Stage IIIA disease tends to be neither as clearly resectable as earlier stages of NSCLC, nor has it reached the point of distant metastasis in which resection has historically been rarely considered (1). As defined by the American Joint Committee on Cancer (AJCC) TNM staging system, IIIA NSCLC includes T3N1M0, T4N0M0, T4N1M0, T1N2M0, and T2N2M0 disease (1). The variability in treatment decision-making may result from the inclusion of a vastly heterogenous population of disease. Management of N2 disease, which is defined as ipsilateral mediastinal nodal involvement, 
is particularly complex given the wide-ranging scope of possible presentations. In particular, the role of resection is increasingly debated in this group. Occult N2 disease can only be identified after pathologic evaluation and is amenable to resection, while bulky N2 disease is clearly evident on imaging and is largely considered to be unresectable. Between these extremes lies the discrete and potentially resectable disease (2). The classic Andre study proposed the organization of resectable $\mathrm{N} 2$ disease into homogenous subgroups based on prognosis and found that minimal N2 disease with one level of nodal involvement had a prognosis similar to stage IIB disease, while both minimal $\mathrm{N} 2$ disease with multiple levels of nodal involvement and clinical N2 disease had prognoses similar to stage IIIB disease (3).

The investigation into the optimal management of stage IIIA NSCLC began decades ago, at a time when the survival rate of patients with resectable disease undergoing standard surgical treatment was low (4). The classic trials by Roth and Rosell were among the first to explore the topic, finding that the addition of preoperative chemotherapy improved the survival of patients compared to surgery alone $(4,5)$. Although still limited by a lack of robust evidence today, medical associations have issued guidelines for the management of stage IIIA NSCLC. The American College of Chest Physicians (ACCP) published the third edition of their clinical practice guidelines in 2013 (6). Of note, the ACCP recommends complete resection of both the primary tumor and affected lymph nodes for occult N2 disease and either definitive chemoradiation or induction therapy followed by surgery for discrete N2 disease. The investigators also recommend systematic nodal sampling or complete nodal dissection for patients undergoing resection (6). The European Society for Medical Oncology (ESMO) published its own consensus guidelines in 2015 (7). ESMO refrained from making specific recommendations but provided the options of induction chemotherapy followed by surgery, induction chemoradiotherapy followed by surgery, and concurrent definitive chemoradiotherapy for managing potentially resectable stage IIIA-N2 disease. In line with ACCP, ESMO also recommended systematic nodal exploration for candidates undergoing resection (7). Given the complexity of presentation and approaches, developing standardized definitions and management algorithms for stage IIIA NSCLC clearly remains a challenge yet to be settled.

There is no clear consensus among thoracic surgeons in the United States when it comes to the surgical management of stage IIIA NSCLC. In microscopic N2 disease, $84 \%$ of surveyed thoracic surgeons favored the use of induction therapy followed by surgery as the treatment of choice (8). When medical oncologists were surveyed, $92 \%$ chose to incorporate surgery into the treatment of single station N2 disease identified on mediastinoscopy, with the most favored option being neoadjuvant chemotherapy followed by surgery and adjuvant chemoradiotherapy (9).

While the majority of thoracic surgeons believe there is a role for surgery in the management of macroscopic stage IIIA NSCLC, there remains a lack of unanimity in regard to factors such as the importance of downstaging and the extent of resection itself; $81 \%$ of surveyed thoracic surgeons favored induction therapy followed by surgery for macroscopic, single station N2 disease with $62 \%$ offering it following evidence of downstaging and the remaining $19 \%$ doing so regardless of disease clearance. The diversity of opinion is greater in managing patients with more advanced disease. For macroscopic, single station $\mathrm{N} 2$ disease with a tumor requiring pneumonectomy, $62 \%$ of surgeons favored induction therapy followed by surgery in the event of downstaging with $30 \%$ electing to perform a pneumonectomy and the remaining $32 \%$ opting for a lobectomy if technically possible (8). In the treatment of bulky, multi-station N2 disease, a majority of medical oncologists surveyed elected to use definitive chemoradiation, with only $48 \%$ including surgery in the management plan (9).

Evidence-based guidelines and theoretical scenarios show a preference for using induction therapy prior to operation; however, the use of such therapy tends to be less wide-spread in clinical practice. Review of data from The Society of Thoracic Surgeons General Thoracic Surgery Database (STS-GTSD) demonstrated that $54 \%$ of patients who underwent operations for clinical stage IIIA-N2 disease were directly treated with surgical resection while the remaining $46 \%$ received induction therapy (10). This study found that while patients in the upfront surgical group achieved 5 -year survival rates of $36 \%$ compared to $35 \%$ for those undergoing induction therapy, improvements could be made in implementing induction therapy as per current guidelines and using invasive mediastinal exploration to avoid overstaging (10).

\section{Benefits of surgery}

Individual patient characteristics influence the benefits 
conferred by surgical management of stage IIIA NSCLC. Disparities exist in the receipt of surgery for this patient population and may be exacerbated by the absence of definitive practice guidelines. Analysis of patient characteristics in the National Cancer Database showed that patients with higher income levels, private insurance, residency in New England, and treatment at an academic center were more likely to have surgical resection included as a modality in the management of their stage IIIA NSCLC (11). Those who were treated at academic medical centers were more likely to be younger, female, non-Caucasian, have fewer comorbidities, and have traveled from far distances to receive treatment (12). Patients who underwent resection at academic centers saw higher rates of induction therapy, more extensive pathologic nodal staging, lower 30-day mortality, and longer median survival when compared to those at non-academic sites (12). Academic centers are known for their large volumes of both patients and skilled surgeons, and they may be able to offer more specialized care than their non-academic counterparts. A review of patients undergoing lung resection in the national Medicare database showed that operative mortality rates were lowest when performed by cardiothoracic and thoracic surgeons, irrespective of hospital volume (13). These operations are complex in nature, and such findings highlight the importance of a highly-skilled multidisciplinary team and adherence to best-practice guidelines in improving postoperative outcomes.

Disease-related factors further influence the benefit of surgical resection and must be considered in the design of an optimal treatment strategy. The Andre study characterized pre-operative $\mathrm{N} 2$ status and levels of nodal involvement as relevant prognostic factors for resectable stage IIIA-N2 NSCLC, with lower survival rates in those with clinically apparent N2 disease and multiple levels of lymph node involvement (3). The histology of the tumor may also impact outcomes, with improved survival rates shown in squamous cell over non-squamous cell histology (14). Analysis of treatment outcomes in resectable N1 NSCLC found that high levels of apoptosis and mitosis predicted better survival for squamous cell subtypes but worse survival and incidence of distant metastasis for adenocarcinoma and large-cell subtypes (15). Response to induction or neoadjuvant therapy also correlates with better survival, with a tumor size reduction of $>36 \%$ on imaging following neoadjuvant concurrent chemoradiation therapy shown to be an independent predictor of longer survival (16). The role of downstaging as a predictive factor has not been defined due to the lack of an accurate restaging modality following induction therapy, but one study has shown no significant difference in overall survival when comparing patients who were downstaged from $\mathrm{N} 2$ to $\mathrm{N} 1 / \mathrm{N} 0$ to those who were not $(16,17)$.

The benefit-and feasibility-of surgery can be difficult to assess in patients with T4 IIIA NSCLC, as it often involves the invasion of disease into local structures. The involvement of mediastinal lymph nodes in N2 disease was found to significantly worsen prognosis following surgery in T4 NSCLC, with other factors such as tumor subtype and use of neoadjuvant or adjuvant therapy playing nonsignificant roles (18). The 5-year survival rate of patients with T4 N0/N1 following surgery has been reported as $43 \%$ (19). Given that stage IIIA NSCLC includes only T4 N0/N1 disease, surgery may be an appropriate treatment modality if patients are able to tolerate the operation. These findings additionally highlight the importance of a thorough investigation into mediastinal nodal staging to guide patient selection. Extensive resections carry a high mortality risk, making it imperative that only patients with favorable disease conditions be selected (20). Complete resection is possible in certain tumors, such as those invading the trachea or carina, with a favorable 5-year mean survival (20). However, tumors that have associated malignant pleural effusions or those that invade the esophagus and vertebrae are largely considered to be unresectable (20). In addition to nodal status and complete resection, invasion of the subclavian artery has been shown to influence survival as those with invasion of the artery have significantly lower 5 -year survival rates than those without (19). The role of induction therapy in $\mathrm{T} 4$ disease has also been investigated. In T3-T4/N0 NSCLC, there was no statistically significant difference in long-term survival between multimodal therapy with induction therapy and surgery alone (21). However, patients who achieved pathological downstaging after induction therapy did have significantly higher overall survival rates than those who did not (21). While these findings provide more insight into the optimal management of T4 IIIA NSCLC, it is important to note that the heterogeneity and relatively small population size of this disease subgroup act as a barrier to attaining substantial evidence that can guide decision-making.

Treatment-related factors such as the type of resection performed also influence overall survival. The North American Intergroup (INT) 0139 phase III trial showed that overall survival was improved in patients who underwent lobectomy after chemoradiation versus 
chemoradiation alone; the same was not true for those undergoing pneumonectomies (22). There are direct relationships between the extent of resection and both the risk of post-operative complications and associated mortality (14). A review of patients with potentially resectable NSCLC undergoing thoracotomy at Memorial Sloan-Kettering Center found the mortality of lobectomies and pneumonectomies to be $2.4 \%$ and $11.3 \%$, respectively (23). The INT 0139 trial also found a high post-operative death rate following pneumonectomies, mostly related to acute respiratory distress syndrome and other respiratory complications (22). Low FEV1, rightsided pneumonectomy, extended resection, and increased blood loss have also been reported as significant factors that increase the risk of post-operative complications (23). When assessing outcomes among operative techniques, other prognostic factors may play a substantial role in the resulting mortality rates. Patients who undergo pneumonectomies have involvement of different lobes or centralized bronchovascular structures, meaning their disease is more extensive than those who undergo lobectomies (24). However, several studies have reported more favorable post-operative mortality rates following pneumectomies, including a single-institution study with a rate of $6.8 \%$ and a review of the STS-GTSD with a rate of $5.6 \%(25,26)$. These studies also identified predictors of mortality including male sex, age over 65 , the presence of congestive heart failure, and the development of bronchopleural fistulas as contributing to higher mortality rates $(25,26)$. As previously mentioned, the specialization of the surgeon performing the operation may also affect patient outcomes. Mortality rates following pneumonectomies have been reported as $11.8 \%$ when performed by thoracic surgeons and $20.2 \%$ when performed by general surgeons, although this difference was not significant (27). While pneumonectomies have a considerable and relatively higher mortality risk compared to that of less extensive resections, there remains significant variation in the predictors and prognostic factors that influence such risk, including an individual surgeon's or institution's experience. In recent years, practice has shifted toward using video-assisted thoracoscopic surgery (VATS) over the thoracotomy approach. Overall survival rates for VATS were significantly higher than for thoracotomy (56.6\% vs. $31.4 \%)$, but no significant difference in recurrence-free survival between the two approaches (17). With the development of new operative techniques comes the opportunity to improve and optimize survival in even the most complex of patients, but it remains prudent to assess their benefit in relation to existing methods.

The advantages of surgery extend beyond that of recurrence and survival. Quality of life, aligned with individual patient beliefs and values, also plays an important role in the determination of which treatment modalities to employ. A study of stage IIIA NSCLC patients in the National Cancer Database receiving either definitive chemoradiotherapy or a trimodal combination of chemotherapy, radiotherapy, and surgical resection was conducted to assess the cost-effectiveness of these management options. The study found an incremental cost increase of $\$ 14,722$ in definitive chemoradiation therapy when compared to the trimodality therapy option (28). The cost of trimodality therapy is also lower when offered at academic centers versus non-academic centers (28). There is a lack of routinely collected data in other quality of life measures for the patient population at hand. As patients experience the effects of their treatment long after they leave the operating room or complete their therapy, further investigation of measures that provide context to the individuals being treated is warranted in order to refine and personalize the therapeutic decision-making process.

\section{The role of surgery in multimodal therapy}

The management of stage IIIA NSCLC is composed of three modalities: systemic therapy, surgical resection, and radiation therapy. The present topic of debate is to what extent and combination these modalities should be implemented. Several prospective, multi-institutional trials have been performed to assess the optimal approach. The INT 0139 trial showed improved progression-free survival in patients undergoing surgery after concurrent chemoradiation therapy, with no significant improvement in overall survival (22). The trial identified either definitive chemoradiation or chemoradiation followed by surgery, with a preference for lobectomy, as options for managing stage IIIA-N2 NSCLC (22). In the European Organisation for Research and Treatment of Cancer (EORTC) 08941 trial, patients with stage IIIA-N2 NSCLC who responded to induction chemotherapy were randomly assigned to receive either surgery or radiotherapy (29). This trial found that median survival time and overall survival rates were 17.5 months and $14 \%$ for the radiotherapy group and 16.4 months and $15.7 \%$ for the surgery group, respectively (29). As a result, the investigators recommended radiotherapy following induction chemotherapy as the 
treatment of choice due to the lack of improved survival and higher morbidity and mortality associated with surgery (29). Although findings across the INT and EORTC studies may vary due to different entry criteria, critique of the EORTC trial involves the fact that induction chemotherapy followed by radiation therapy is not considered a standard method of treatment (22). A trial by the SAKK Lung Cancer Project Group calls into question the necessity of trimodal therapy, as the addition of radiotherapy to induction chemotherapy prior to surgery did not result in an improvement in eventfree or overall survival (30). Instead, the investigators recommend the use of induction chemotherapy in combination with one definitive local treatment, either surgery or radiotherapy, in the management of resectable non-superior sulcus tumors (30). The Southwest Oncology Group (SWOG) 9416 trial assessed the feasibility of induction chemoradiotherapy followed by resection in $\mathrm{T} 3$ and T4 superior sulcus NSCLC and found high rates of local control and long-term survival, which were improved from those seen when radiation alone was followed by surgery (31). These trials all limited the radiation used to 45 Gy due to concerns over increasing operative morbidity with higher doses of radiation, yet no increases in postoperative morbidity and mortality have been shown when comparing trimodal therapy with high-dose 60 Gy radiation to that with standard-dose $45 \mathrm{~Gy}$ (32). As higher radiation doses are associated with better local tumor control and mediastinal nodal clearance, these findings are promising in terms of enhancing induction therapy and allowing for greater benefit of the resection that follows (32).

The question of bimodality versus trimodality therapy use in stage IIIA NSCLC has also been addressed in smaller single-institution studies, comprehensive database reviews, and meta-analyses. One single-institution study found better overall survival, progression-free survival, and loco-regional control in trimodality therapy versus definitive concurrent chemoradiation, although these benefits were not conferred to those with either subcarinal nodal or extensive mediastinal involvement (33). Review of the Surveillance, Epidemiology, and End Results (SEER) database showed an increase in the proportion of patients treated with a combination of surgery and radiation as opposed to radiation alone from 2002 to 2012, with a significantly improved overall survival in those receiving combination therapy (34). A meta-analysis of six trials found no difference in overall survival when either surgery or radiation were used in bimodality regimens with chemotherapy, with a $13 \%$ improvement in survival when surgery was used in a trimodality regimen with chemoradiotherapy (35). This gain in survival was not statistically significant but favored the use of a trimodal treatment regimen over a bimodal one (35).

Much of the discussion of the surgical role in managing stage IIIA NSCLC centers around the notion of surgical resection as an upfront modality used in advance of either chemotherapy or radiation therapy. Surgery may also be employed in the form of a salvage resection, which follows the failure of initial therapy such as definitive concurrent chemoradiation. Salvage operations have been shown to result in variable operative times, major complications, and considerable overall morbidity (36). Review of the National Cancer Database showed a 5 -year survival rate of $38.5 \%$ for patients with stage IIIA clinical N2 NSCLC undergoing definitive chemoradiation followed by salvage resection and $46.6 \%$ for those undergoing early surgery as part of trimodality therapy (37). A study at the Saitama Cancer Center found similar results across various stages of NSCLC, with a 5 -year survival rate of $65.2 \%$ in patients undergoing induction therapy followed by surgery and $62.2 \%$ in those undergoing salvage surgery (38). With a lack of significant difference in these survival rates, the benefit of having patients endure an extended period of chemoradiation only to undergo a complicated resection in the end must be reassessed in those who can tolerate upfront resection.

While the benefit of induction therapy prior to surgical resection has been established, there remains the question of how adjuvant and post-operative therapies can be implemented in the management of resectable stage IIIA NSCLC as well. The Adjuvant Navelbine International Trialist Association (ANITA) trial is a randomized controlled study that looked at the effect of adjuvant chemotherapy on survival in completely resected stage IB, II, and IIIA NSCLC (39). The ANITA trial found improved median survival, reduced risk for death, and higher overall survival rates in the group of patients receiving adjuvant vinorelbine plus cisplatin chemotherapy compared to the control group (39). A subgroup analysis of the ANITA trial also found improved survival when post-operative radiation therapy was used in patients with pathologic N2 disease but worsened survival in those with pathologic N1 disease, all following adjuvant chemotherapy (40). These findings highlight the continuing need for not only defining the appropriate combination of treatment modalities, but also determining the optimal timeline in which to administer one in relation to another. 


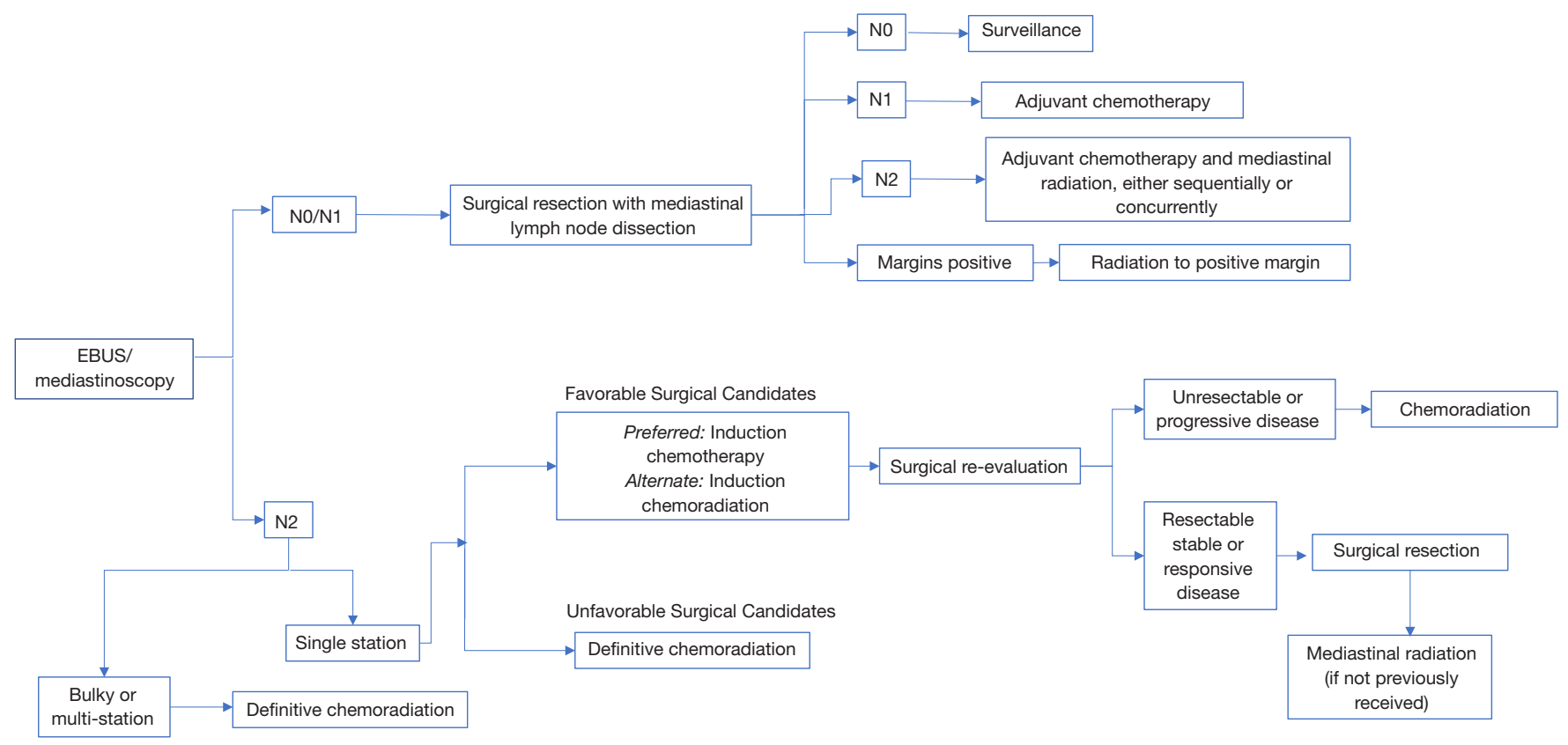

Figure 1 Recommendations for management of N2 IIIA non-small cell lung cancer. EBUS, endobronchial ultrasound.

\section{Our experience}

At our institution, a practice algorithm has been developed on the basis of the specific patient population and services offered at MD Anderson Cancer Center (41). Figure 1 shows an adaptation of the published algorithm for initial and adjuvant treatment of stage IIIA clinical N2 NSCLC. Once clinical N2 disease status has been identified via MRI brain, PET scan, and pulmonary function tests, pretreatment evaluation includes the use of bronchoscopy, endobronchial ultrasound-guided fine-needle aspiration (EBUS-FNA), or mediastinoscopy in order to determine nodal involvement. Should N2 nodes be histologically negative, surgical resection with mediastinal lymph node dissection is performed and the nodes are pathologically evaluated. If nodal status is then determined to be N2-3, post-operative adjuvant chemotherapy followed by radiation therapy, either alone or concurrent chemoradiation, is the treatment of choice. Should N2 nodes be positive, induction chemotherapy is offered followed by surgical re-evaluation if the patients are thought to be favorable candidates for surgery in terms of anatomic resectability, performance status, and ability to tolerate an operation. Upon reevaluation, if the patients remain surgical candidates, they undergo resection followed by adjuvant radiation. While we tend to favor trimodal therapy with induction chemotherapy, then surgery, followed by radiation, we do recognize induction chemoradiation followed by surgery as an acceptable alternative. Furthermore, for patients that are declared not to be surgical candidates upfront, definitive chemoradiation is the therapy of choice. Finally, for those patients who are considered possible surgical candidates and either progress during induction chemotherapy or are no longer felt to be appropriate candidates for surgery, we offer chemoradiation after induction chemotherapy. The assessment of favorable surgical candidacy is challenging in N2 disease, particularly when the categorization of subtypes lacks standardized definition. While occult or single-level disease is considered more favorable than bulky or multilevel disease, it is difficult to assign surgical candidacy from these definitions alone $(2,3)$. Ultimately, the decision to deem N2 disease resectable must be made from the clinical judgment of the multi-disciplinary team involved in the case on the basis of factors previously mentioned.

We recommend that resectability be determined by thoracic surgical oncologists and that all patients considered for surgical resection undergo appropriate pulmonary function testing. Additionally, we believe lobectomy and pneumonectomy to be the operations of choice in the surgical management of NSCLC, with lobectomy being preferred if negative margins can be attained. Pneumonectomy should be avoided if at all possible, with 
the use of sleeve resections when feasible. All patients should be discussed in a multidisciplinary fashion, and for patients requiring extensive resection, presentation at a thoracic oncology tumor board is highly recommended. Limited resections are also acceptable in those who cannot tolerate anatomic resection. We advocate for complete N1 and N2 nodal dissection in all patients undergoing surgical resection.

\section{Future of surgery in the face of emerging therapeutic options}

As advancements are made in the surgical realm, parallel achievements have been reached in medical therapy as well. While emerging therapeutic options hold promise, they may also serve to further complicate the development of a treatment algorithm composed of various modalities. Immunotherapy in particular has been a topic of recent interest, with the use of agents such as Nivolumab and Ipilimumab being studied in resectable NSCLC. Nivolumab, a monoclonal antibody to the programmed cell death protein 1 checkpoint receptor (PD-1), did not result in unexpected post-operative morbidity or mortality in comparison to chemotherapy when used as a neoadjuvant agent in resectable stage I to IIIA NSCLC (42). Ipilimumab, a monoclonal antibody to cytotoxic T-lymphocyte-associated protein 4 (CTLA-4), has been shown to improve progression-free survival in phased combination with chemotherapy for advanced untreated NSCLC without impacting the toxicities seen with chemotherapy alone (43). A review of the TOP1201 trial assessed the feasibility of a combination of neo-adjuvant chemotherapy and Ipilimumab followed by surgery for stage II-IIIA NSCLC (44). The study did not find an increase in adverse surgical outcomes or negative effect on post-operative overall survival for patients receiving neoadjuvant chemotherapy and Ipilimumab when compared to patients receiving chemotherapy alone (44). A high response rate has been shown when a combination of Nivolumab and Ipilimumab was used as first-line therapy for patients with NSCLC tumors that express programmed death-ligand 1 (PD-L1) (45). In locally advanced NSCLC that was deemed unresectable, treatment with the anti-PD-L1 monoclonal antibody Durvalumab showed a significantly longer progression-free survival time when compared to definitive chemoradiation therapy (46).

In the subgroup of patients with epidermal growth factor receptor (EGFR) mutation positive stage IIIA NSCLC, the use of EGFR tyrosine kinase inhibitors such as erlotinib and gefitinib holds considerable promise. The Chinese Thoracic Oncology Group (CTONG) 1103 trial showed median progression-free survival to be significantly longer when erlotinib was used as neo-adjuvant/adjuvant therapy versus gemcitabine plus cisplatin chemotherapy (47). The EVAN trial indicated that 2-year disease freesurvival was significantly longer with adjuvant erlotinib compared to chemotherapy in patients with resected EGFR mutation positive disease (48). Similarly, the ADJUVANT/ CTONG1104 trial has shown significantly longer disease-free survival with adjuvant Gefitinib compared to vinorelbine plus cisplatin chemotherapy in resected stage II-IIIA disease (49). The exploration of therapy targeted to the EGFR mutation may very well pave the way for treatment modalities that target other mutations and disease subpopulations, allowing for a more personalized approach to disease management. As more data become available and the use of immunotherapy and molecular targeted therapy becomes widespread, the already-complicated process of determining a definitive treatment algorithm will become increasingly more complex. The promising results following the incorporation of these therapies into management of NSCLC warrant further investigation through clinical trials in order to establish efficacy and determine the optimal combination of treatment modalities based on the options available.

\section{Final considerations}

Stage IIIA NSCLC covers a heterogenous population of disease in which neither the definition of subpopulations nor optimal management have yet to be determined. As improvements in the modalities available are developed and advancements in medical therapy are discovered, the combination and extent of such modalities continue to be studied. While several prospective trials have been conducted, the role of surgery in particular has never been clearly established. The disconnect between the evidence available and the failure to develop a coherent therapeutic algorithm lies in the heterogeneity of disease studied as well as individual patient and tumor characteristics that need further investigation. It is clear that there lies a benefit in incorporating surgery into the treatment of stage IIIA NSCLC, but the question that still needs answering is which subpopulations the surgery should be offered to. Without more randomized prospective clinical trials with clearly defined and standardized inclusion criteria, 
management algorithms cannot be founded with supporting evidence. Finally, the role of surgery must be reimagined with new and evolving modalities in mind.

\section{Acknowledgments}

Funding: None.

\section{Footnote}

Provenance and Peer Review: This article was commissioned by the Guest Editors (Fabrizio Minervini and Marco Scarci) for the series "Controversies in the Management of Stage IIIA Non-Small-Cell Lung Cancer" published in Current Challenges in Thoracic Surgery. The article has undergone external peer review.

Peer Review File: Available at https://ccts.amegroups.com/ article/view/10.21037/ccts-20-65/prf

Conflicts of Interest: All authors have completed the ICMJE uniform disclosure form (available at https://ccts. amegroups.com/article/view/10.21037/ccts-20-65/coif). The series "Controversies in the Management of Stage IIIA Non-Small-Cell Lung Cancer" was commissioned by the editorial office without any funding or sponsorship. The authors have no other conflicts of interest to declare.

Ethical Statement: The authors are accountable for all aspects of the work in ensuring that questions related to the accuracy or integrity of any part of the work are appropriately investigated and resolved.

Open Access Statement: This is an Open Access article distributed in accordance with the Creative Commons Attribution-NonCommercial-NoDerivs 4.0 International License (CC BY-NC-ND 4.0), which permits the noncommercial replication and distribution of the article with the strict proviso that no changes or edits are made and the original work is properly cited (including links to both the formal publication through the relevant DOI and the license). See: https://creativecommons.org/licenses/by-nc-nd/4.0/.

\section{References}

1. Van Schil PE, Berzenji L, Yogeswaran SK, et al. Surgical Management of Stage IIIA Non-Small Cell Lung Cancer. Front Oncol 2017;7:249.
2. Evison M, Clive A, Castle L, et al. Resectable Clinical N2 Non-Small Cell Lung Cancer; What Is the Optimal Treatment Strategy? An Update by the British Thoracic Society Lung Cancer Specialist Advisory Group. J Thorac Oncol 2017;12:1434-41.

3. Andre F, Grunenwald D, Pignon JP, et al. Survival of patients with resected $\mathrm{N} 2$ non-small-cell lung cancer: evidence for a subclassification and implications. J Clin Oncol 2000;18:2981-9.

4. Roth JA, Fossella F, Komaki R, et al. A randomized trial comparing perioperative chemotherapy and surgery with surgery alone in resectable stage IIIA non-small-cell lung cancer. J Natl Cancer Inst 1994;86:673-80.

5. Rosell R, Gomez-Codina J, Camps C, et al. A randomized trial comparing preoperative chemotherapy plus surgery with surgery alone in patients with non-small-cell lung cancer. N Engl J Med 1994;330:153-8.

6. Ramnath N, Dilling TJ, Harris LJ, et al. Treatment of stage III non-small cell lung cancer: Diagnosis and management of lung cancer, 3 rd ed: American College of Chest Physicians evidence-based clinical practice guidelines. Chest 2013;143:e314S-e340S.

7. Eberhardt WE, De Ruysscher D, Weder W, et al. 2nd ESMO Consensus Conference in Lung Cancer: locally advanced stage III non-small-cell lung cancer. Ann Oncol 2015;26:1573-88.

8. Veeramachaneni NK, Feins RH, Stephenson BJ, et al. Management of stage IIIA non-small cell lung cancer by thoracic surgeons in North America. Ann Thorac Surg 2012;94:922-6; discussion 926-8.

9. Tanner NT, Gomez M, Rainwater C, et al. Physician preferences for management of patients with stage IIIA NSCLC: impact of bulk of nodal disease on therapy selection. J Thorac Oncol 2012;7:365-9.

10. Boffa D, Fernandez FG, Kim S, et al. Surgically Managed Clinical Stage IIIA-Clinical N2 Lung Cancer in The Society of Thoracic Surgeons Database. Ann Thorac Surg 2017;104:395-403.

11. Trifiletti DM, Zaorsky NG, Grover S, et al. Surgical Resection of Stage IIIA Non-Small Cell Lung Cancer in the United States. Int J Radiat Oncol Biol Phys 2017;99:E418.

12. Samson P, Patel A, Crabtree TD, et al. Multidisciplinary Treatment for Stage IIIA Non-Small Cell Lung Cancer: Does Institution Type Matter? Ann Thorac Surg 2015;100:1773-9.

13. Goodney PP, Lucas FL, Stukel TA, et al. Surgeon specialty and operative mortality with lung resection. Ann Surg 
2005;241:179-84.

14. Birim O, Kappetein AP, van Klaveren RJ, et al. Prognostic factors in non-small cell lung cancer surgery. Eur J Surg Oncol 2006;32:12-23.

15. Komaki R, Fujii T, Perkins P, et al. Apoptosis and mitosis as prognostic factors in pathologically staged N1 nonsmall cell lung cancer. Int J Radiat Oncol Biol Phys 1996;36:601-5.

16. Lim HJ, Lee HY, Lee KS, et al. Predictive factors for survival in stage IIIA N2 NSCLC patients treated with neoadjuvant CCRT followed by surgery. Cancer Chemother Pharmacol 2015;75:77-85.

17. Yang CF, Adil SM, Anderson KL, et al. Impact of patient selection and treatment strategies on outcomes after lobectomy for biopsy-proven stage IIIA pN2 non-small cell lung cancer. Eur J Cardiothorac Surg 2016;49:1607-13.

18. Lucchi M, Viti A, Melfi F, et al. IIIB-T4 non-small cell lung cancer: indications and results of surgical treatment. J Cardiovasc Surg (Torino) 2007;48:369-74.

19. Yildizeli B, Dartevelle PG, Fadel E, et al. Results of primary surgery with $\mathrm{T} 4$ non-small cell lung cancer during a 25-year period in a single center: the benefit is worth the risk. Ann Thorac Surg 2008;86:1065-75; discussion 1074-5.

20. Pitz CC, Brutel de la Riviere A, van Swieten HA, et al. Results of surgical treatment of T4 non-small cell lung cancer. Eur J Cardiothorac Surg 2003;24:1013-8.

21. Lococo F, Cesario A, Margaritora S, et al. Induction therapy followed by surgery for T3-T4/N0 non-small cell lung cancer: long-term results. Ann Thorac Surg 2012;93:1633-40.

22. Albain KS, Swann RS, Rusch VW, et al. Radiotherapy plus chemotherapy with or without surgical resection for stage III non-small-cell lung cancer: a phase III randomised controlled trial. Lancet 2009;374:379-86.

23. Martin J, Ginsberg RJ, Abolhoda A, et al. Morbidity and mortality after neoadjuvant therapy for lung cancer: the risks of right pneumonectomy. Ann Thorac Surg 2001;72:1149-54.

24. Perentes JY, Zellweger M, Gonzalez M. Is pneumonectomy still necessary? J Thorac Dis 2018;10:6414-7.

25. Alexiou C, Beggs D, Rogers ML, et al. Pneumonectomy for non-small cell lung cancer: predictors of operative mortality and survival. Eur J Cardiothorac Surg 2001;20:476-80.

26. Shapiro M, Swanson SJ, Wright CD, et al. Predictors of major morbidity and mortality after pneumonectomy utilizing the Society for Thoracic Surgeons General Thoracic Surgery Database. Ann Thorac Surg 2010;90:927-34; discussion 934-5.

27. Silvestri GA, Handy J, Lackland D, et al. Specialists achieve better outcomes than generalists for lung cancer surgery. Chest 1998;114:675-80.

28. Samson P, Patel A, Robinson CG, et al. The Role of Surgical Resection in Stage IIIA Non-Small Cell Lung Cancer: A Decision and Cost-Effectiveness Analysis. Ann Thorac Surg 2015;100:2026-32; discussion 2032.

29. van Meerbeeck JP, Kramer GW, Van Schil PE, et al. Randomized controlled trial of resection versus radiotherapy after induction chemotherapy in stage IIIA-N2 non-small-cell lung cancer. J Natl Cancer Inst 2007;99:442-50.

30. Pless M, Stupp R, Ris HB, et al. Induction chemoradiation in stage IIIA/N2 non-small-cell lung cancer: a phase 3 randomised trial. Lancet 2015;386:1049-56.

31. Rusch VW, Giroux DJ, Kraut MJ, et al. Induction chemoradiation and surgical resection for superior sulcus non-small-cell lung carcinomas: long-term results of Southwest Oncology Group Trial 9416 (Intergroup Trial 0160). J Clin Oncol 2007;25:313-8.

32. Seder CW, Allen MS, Cassivi SD, et al. Stage IIIA nonsmall cell lung cancer: morbidity and mortality of three distinct multimodality regimens. Ann Thorac Surg 2013;95:1708-16.

33. Shepherd AF, Leeman JE, Wild A, et al. A Comparison of Trimodality Therapy Versus Definitive Concurrent Chemoradiation in Patients With Stage IIIA Nonsmall Cell Lung Cancer. Int J Radiat Oncol Biol Phys 2017;99:E495-E496.

34. Smith AW, Chitti BS, Wernicke AG, et al. Increasing Use of Combination Surgery and Radiation Compared to Surgery or Radiation Alone Has Improved Survival in Patients With Stage IIIA Non-Small Cell Lung Cancer: A Population-Based Study of Patterns of Care. Int J Radiat Oncol Biol Phys 2016;96:E468.

35. McElnay PJ, Choong A, Jordan E, et al. Outcome of surgery versus radiotherapy after induction treatment in patients with N2 disease: systematic review and metaanalysis of randomised trials. Thorax 2015;70:764-8.

36. Petrella F, Leo F, Veronesi G, et al. "Salvage" Surgery for Primary Mediastinal Malignancies: Is it Worthwhile? J Thorac Oncol 2008;3:53-8.

37. Ye JC, Ding L, Atay SM, et al. Comparable Survival Between Upfront Tri-Modality Therapy and Definitive Chemoradiation Therapy Requiring Salvage Resection in 
cN2 Stage IIIA Non-Small Cell Lung Cancer. Int J Radiat Oncol Biol Phys 2018;102:E717.

38. Uramoto H, Nakajima Y, Kinoshita H, et al. Equivalent Outcome of Patients with Locally Advanced NSCLC Treated with Salvage Surgery Compared to Induction Chemotherapy Followed by Surgical Resection. Anticancer Res 2016;36:4243-7.

39. Douillard JY, Rosell R, De Lena M, et al. Adjuvant vinorelbine plus cisplatin versus observation in patients with completely resected stage IB-IIIA non-small-cell lung cancer (Adjuvant Navelbine International Trialist Association [ANITA]): a randomised controlled trial. Lancet Oncol 2006;7:719-27.

40. Douillard JY, Rosell R, De Lena M, et al. Impact of postoperative radiation therapy on survival in patients with complete resection and stage I, II, or IIIA non-smallcell lung cancer treated with adjuvant chemotherapy: the adjuvant Navelbine International Trialist Association (ANITA) Randomized Trial. Int J Radiat Oncol Biol Phys 2008;72:695-701.

41. Non-Small Cell Lung Cancer [Internet]. Houston: The University of Texas MD Anderson Cancer Center; c2017. [cited 7 July 2020]. Available online: https://www. mdanderson.org/documents/for-physicians/algorithms/ cancer-treatment/ca-treatment-non-small-cell-webalgorithm.pdf

42. Bott MJ, Yang SC, Park BJ, et al. Initial results of pulmonary resection after neoadjuvant nivolumab in patients with resectable non-small cell lung cancer. J Thorac Cardiovasc Surg 2019;158:269-76.

43. Lynch TJ, Bondarenko I, Luft A, et al. Ipilimumab in

doi: $10.21037 /$ ccts-20-65

Cite this article as: Iranpour N, Olive JK, Antonoff MB. Management of stage IIIA non-small-cell lung cancer: role of surgery. Curr Chall Thorac Surg 2021;3:26. combination with paclitaxel and carboplatin as first-line treatment in stage IIIB/IV non-small-cell lung cancer: results from a randomized, double-blind, multicenter phase II study. J Clin Oncol 2012;30:2046-54.

44. Yang CJ, McSherry F, Mayne NR, et al. Surgical Outcomes After Neoadjuvant Chemotherapy and Ipilimumab for Non-Small Cell Lung Cancer. Ann Thorac Surg 2018;105:924-9.

45. Hellmann MD, Rizvi NA, Goldman JW, et al. Nivolumab plus ipilimumab as first-line treatment for advanced nonsmall-cell lung cancer (CheckMate 012): results of an open-label, phase 1, multicohort study. Lancet Oncol 2017;18:31-41.

46. Antonia SJ, Villegas A, Daniel D, et al. Durvalumab after Chemoradiotherapy in Stage III Non-Small-Cell Lung Cancer. N Engl J Med 2017;377:1919-29.

47. Zhong WZ, Chen KN, Chen C, et al. Erlotinib Versus Gemcitabine Plus Cisplatin as Neoadjuvant Treatment of Stage IIIA-N2 EGFR-Mutant Non-Small-Cell Lung Cancer (EMERGING-CTONG 1103): A Randomized Phase II Study. J Clin Oncol 2019;37:2235-45.

48. Yue D, Xu S, Wang Q, et al. Erlotinib versus vinorelbine plus cisplatin as adjuvant therapy in Chinese patients with stage IIIA EGFR mutation-positive non-small-cell lung cancer (EVAN): a randomised, open-label, phase 2 trial. Lancet Respir Med 2018;6:863-73.

49. Zhong WZ, Wang Q, Mao WM, et al. Gefitinib versus vinorelbine plus cisplatin as adjuvant treatment for stage II-IIIA (N1-N2) EGFR-mutant NSCLC (ADJUVANT/ CTONG1104): a randomised, open-label, phase 3 study. Lancet Oncol 2018;19:139-48. 